\title{
FREE TRANSITION ON A SLENDER CONE IN A QUIET AND A CONVENTIONAL WIND TUNNEL AND THE EFFECT OF ULTRASONICALLY ABSORPTIVE MATERIALS
}

\section{S. Willems ${ }^{1}$, A. Gülhan ${ }^{1}$, C. A. C. Ward ${ }^{2}$, and S. P. Schneider ${ }^{2}$}

\author{
${ }^{1}$ German Aerospace Center (DLR) \\ Supersonic and Hypersonic Technology Department \\ Institute of Aerodynamics and Flow Technology \\ Linder Höhe, Köln 51147, Germany \\ ${ }^{2}$ School of Aeronautics and Astronautics \\ Purdue University \\ West Lafayette, IN 47907-1282, USA
}

\begin{abstract}
Transition from laminar to turbulent hypersonic boundary layers is the topic of several research projects world-wide. Most experimental work is done in conventional wind tunnels, although their free stream turbulence does not match free flight conditions and has a significant influence onto the transition process. Experiments performed with the same 3 degree half angle cone in a conventional and a quiet wind tunnel at Mach 6 justify this approach. The formation, amplification, and decay of the second (Mack) modes is compared based on high-frequency pressure measurements. In addition, damping of these modes with ultrasonically absorptive surfaces was tested.
\end{abstract}

\section{INTRODUCTION}

The transition from a laminar to a turbulent boundary layer is accompanied by an increase of the heat flux and drag. Therefore, it is essential for the design of hypersonic vehicles to predict the transition location correctly. In addition, it is often desirable to delay the transition as long as possible. In nonlifting hypersonic flows over smooth surfaces, the transition is most likely provoked by the first and second mode instabilities. As the first mode (Tollmien-Schlichting 
waves) can be damped by cooled structures, the second mode (Mack mode) becomes dominant. The formation and amplification of the second (Mack) mode is the topic of several research projects. Most of the experiments are performed in classical wind tunnels although the free stream fluctuations have a significant effect onto the transition process. The disturbance level in a quiet wind tunnel is one or two orders of magnitude lower and, therefore, much closer to the conditions at real flight. But there are just a few quiet wind tunnels and their operating envelope is limited. A direct comparison of experiments in a quiet and a conventional wind tunnel is important to identify the drawbacks and opportunities of conventional wind tunnels for hypersonic transition research.

The experiments presented in this paper were performed in the Boeing/AFOSR Mach-6 Quiet Tunnel (BAM6QT) at Purdue University and in the hypersonic wind tunnel $(\mathrm{H} 2 \mathrm{~K})$ of the German Aerospace Center (DLR) in

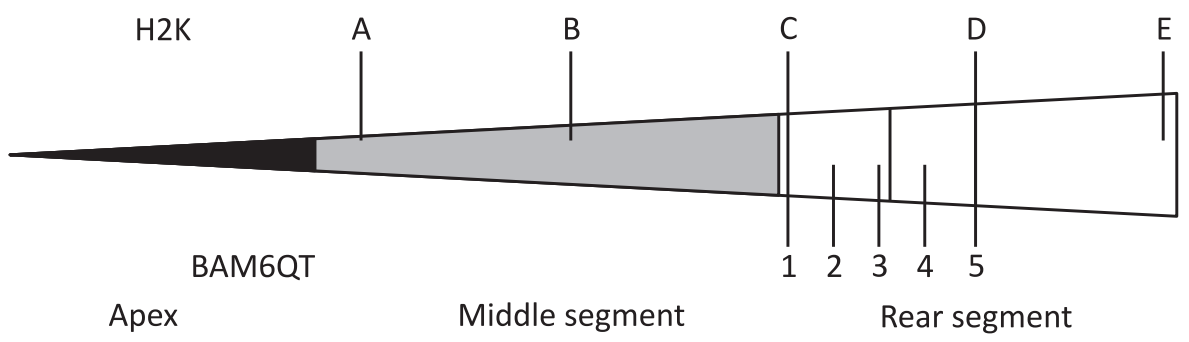

Figure 1 Drawing of the model with segments and marked sensor positions

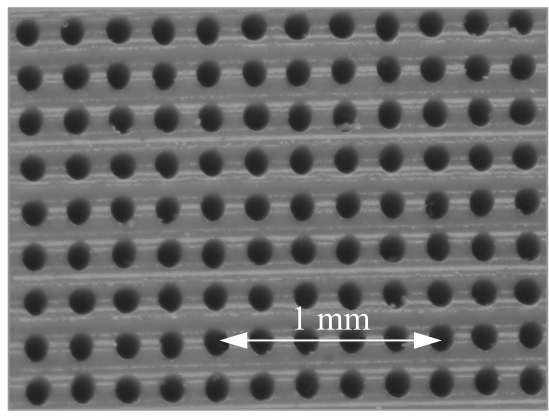

(a)

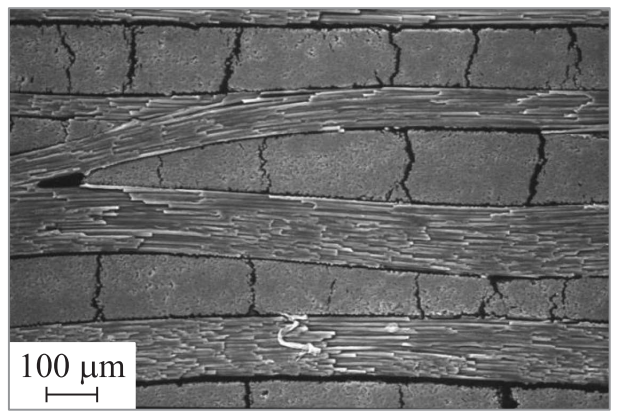

(b)

Figure 2 Details of the ultrasonically absorptive surfaces: (a) PEEK surface with regular holes; and (b) $\mathrm{C} / \mathrm{C}$ surface with random pores 
Cologne. To improve comparability, the same model, sensors, and data acquisition system were used.

There are numerous attempts for passive or active damping of these trapped acoustic waves. Rasheed et al. [1] demonstrated a damping of the second (Mack) modes and a delay of the transition on a 5 degree half cone with a regular porous surface at Mach 5. Fedorov et al. [2] and Wagner et al. [3] verified the damping of these acoustic waves with a 7 degree half cone and a porous coating of random microstructures at Mach 6 . For the transition experiments presented here, a 3 degree half angle cone with either a plain surface, regular holes, or a random porous surface was tested at Mach 6 .

The used model (Figs. 1 and 2) is equipped with PCB sensors for the detection of pressure fluctuations at high frequencies as well as Kulite sensors for those at intermediate and low frequencies. The main model parts are made of polyether ether ketone (PEEK) to enable simultaneous measurements of the transition position with infrared cameras for the $\mathrm{H} 2 \mathrm{~K}$ experiments. For the BAM6QT experiments, the rear segment was replaced with an aluminium segment to use temperature sensitive paint (TSP).

\section{EXPERIMENTAL SETUP}

\subsection{Model}

The basic model shape is a circular cone with 3 degree half angle. The model consists of three exchangeable segments: the apex, the middle segment, and the rear segment, and is supported by a central steel shaft (see Fig. 1). A sharp steel apex with a nose radius below $0.15 \mathrm{~mm}$ was used for all experiments in this paper. Three different middle segments were in use. Two made of PEEK allow quantitative infrared thermography in $\mathrm{H} 2 \mathrm{~K}$ : the first with a plain surface and the second with a generic porous surface formed by regular uniform blind holes. The holes are $80 \mu \mathrm{m}$ in diameter, at least $1000 \mu \mathrm{m}$ in depth, and placed every $200 \mu \mathrm{m}$, thus the porosity is $12.6 \%$. A close-up is shown in Fig. $2 a$. The choice of the hole dimensions originates from the simulations $[4,5]$ with NOLOT and technical feasibility. The Fraunhofer Institute for Laser Technology (ILT) in Aachen performed the manufacturing of these holes with the help of laser drilling [6] using a pulsed INNOSLAB laser. In circumferential direction, one third $\left(120^{\circ}\right)$ of the surface is perforated. The perforated area starts at a radius of $15.5 \mathrm{~mm}$ and ends at a radius of $39.5 \mathrm{~mm}$; thus, the porous area has a length of $456 \mathrm{~mm}$ and contains about 660000 holes. The third middle segment is made of a $\mathrm{C} / \mathrm{C}$ material with a random porosity (see Fig. $2 b$ ) manufactured by the DLR Institute of Structures and Design in Stuttgart. The typical diameter of the pores is 10 to $40 \mu \mathrm{m}$ and the porosity is $12.1 \%$. The rear segment used in $\mathrm{H} 2 \mathrm{~K}$ is made of PEEK and has a base radius of $90 \mathrm{~mm}$. Due to the smaller core of uniform flow in the BAM6QT, two shorter rear segments with base radius 
of 45 and $50 \mathrm{~mm}$ were used. They are made of aluminum, slightly undersized to allow for the application of TSP, since infrared thermography is not possible in the BAM6QT.

\subsection{Quiet Wind Tunnel BAM6QT}

The Boeing/AFOSR Mach 6 Quiet Tunnel (BAM6QT) is a Ludwieg tube with a long driver tube and converging-diverging nozzle for Mach 6. A schematic of the tunnel is shown in Fig. 3. Several features ensure a laminar boundary layer on the nozzle walls. This includes boundary layer suction upstream of the throat, slowly increasing diameter in the divergent section of the nozzle, polished nozzle walls, and the position of the burst diaphragms downstream of the test section. With this, the turbulence level of the free flow in "quiet" mode is of the order of $0.05 \%$. If the bleed lip for the boundary layer suction is closed, the tunnel operates in "noisy" mode with a turbulence level of the

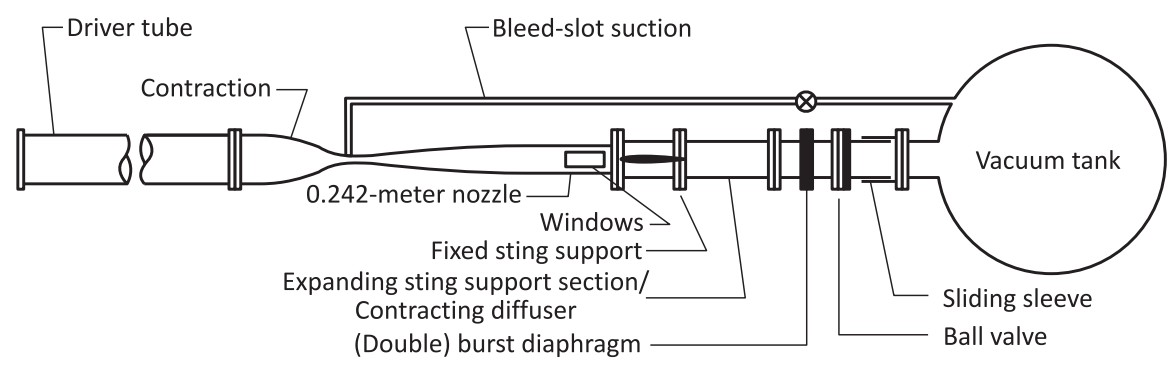

Figure 3 Schematic of Boeing/AFOSR Mach 6 Quiet Tunnel (BAM6QT) [7]

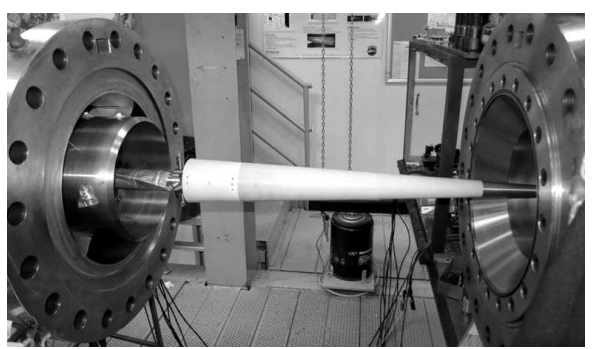

(a)

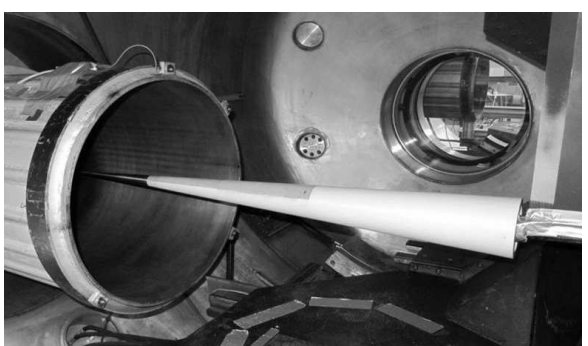

(b)

Figure 4 Pictures of the setups: (a) model inside the BAM6QT; and (b) model inside the $\mathrm{H} 2 \mathrm{~K}$ 
Table 1 Flow conditions for the tests

\begin{tabular}{ccccccc}
\hline Wind tunnel & $\mathrm{Ma}$ & $T_{0}, \mathrm{~K}$ & $p_{0}, \mathrm{kPa}$ & $T_{\infty}, \mathrm{K}$ & $p_{\infty}, \mathrm{Pa}$ & $\mathrm{Re}_{u, \infty}, 10^{6} \mathrm{~m}^{-1}$ \\
\hline \multirow{2}{*}{$\mathrm{H} 2 \mathrm{~K}$} & 6.0 & 590 & 510 & 72 & 323 & 3.2 \\
& 6.0 & 500 & 510 & 61 & 323 & 4.1 \\
\hline \multirow{2}{*}{ BAM6QT noisy } & 5.8 & 422 & 212 & 55 & 165 & 2.4 \\
& 5.8 & 424 & 294 & 55 & 229 & 3.3 \\
\hline \multirow{5}{*}{ BAM6QT quiet } & 6.0 & 427 & 225 & 52 & 143 & 2.3 \\
& 6.0 & 420 & 694 & 51 & 439 & 7.2 \\
& 6.0 & 424 & 928 & 52 & 587 & 9.5 \\
\hline
\end{tabular}

order of $3 \%$. The air in the driver tube is pressurized up to $2 \mathrm{MPa}$ and electrically heated up to $430 \mathrm{~K}$. The length of the driver tube ensures stable flow conditions for about $100 \mathrm{~ms}$ and the stepwise blowdown measurements with several Reynolds numbers in the same run. Figure $4 a$ shows the test section with the model. The inflow conditions of the experiments in BAM6QT are listed in Table 1.

\subsection{Conventional Wind Tunnel H2K}

The hypersonic wind tunnel Cologne $(\mathrm{H} 2 \mathrm{~K})$ is a classical blowdown wind tunnel with a free jet test section and a test time of $30 \mathrm{~s}$. A schematic of the tunnel is shown in Fig. 5. For the experiments, a Mach 6 contoured nozzle with an exit diameter of $600 \mathrm{~mm}$ was used. The test gas air is heated with resistance heaters. Figure $4 b$ shows the test section with the model. For further information about the $\mathrm{H} 2 \mathrm{~K}$, see [8]. The inflow conditions of the experiments in $\mathrm{H} 2 \mathrm{~K}$ are listed in Table 1.

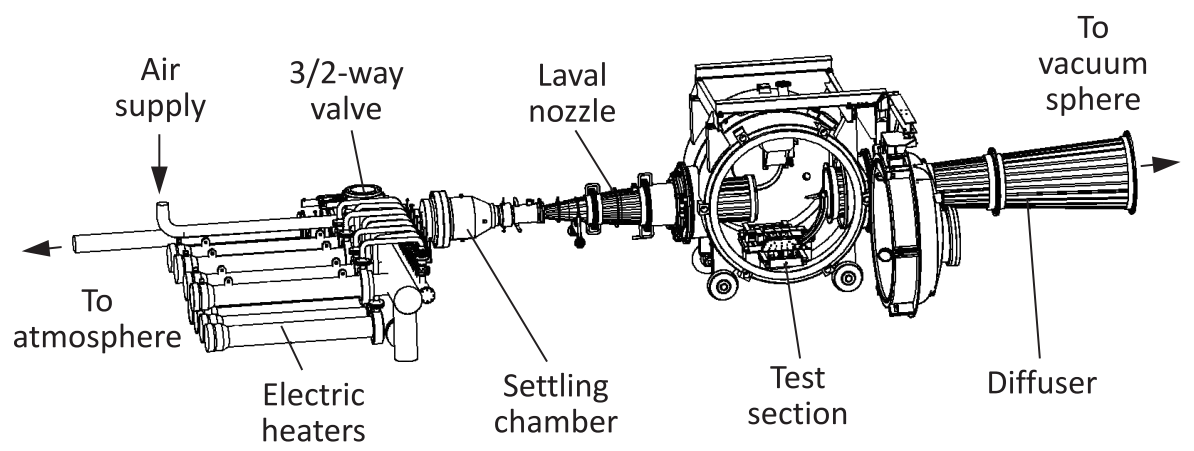

Figure 5 Schematic of hypersonic wind tunnel Cologne (H2K) 


\subsection{Data Acquisition}

For the experiments, the same data acquisition system was used and the same sensors as far as possible. The model is equipped with 8 to $12 \mathrm{PCB}^{\circledR} 132 \mathrm{~A} 31$ sensors with a 350-kilopascal range and a resonant frequency above $1 \mathrm{MHz}$. They are connected to signal conditioners $\mathrm{PCB}^{\circledR} 482 \mathrm{C} 05$ and their output signals are measured with Adlink PXI-9816D/512 digitizers, which enable a 16-bit resolution and a sample rate of $5 \mathrm{MHz}$. Three to five of the PCB sensors are placed on a generatrix numbered according to Fig. 1 with the exact positions given in Table 2. At the positions 1, 3, 5, C, and E, there are 4 PCB sensors around the circumference. For the $\mathrm{H} 2 \mathrm{~K}$ experiments, the model is equipped with 4 Kulite ${ }^{\circledR}$ XCQ-080 B-screen sensors with a 35-kilopascal range and a natural frequency of $150 \mathrm{kHz}$ for static and low frequency surface pressure measurements. They are connected to a NI PXIe-4331 bridge module, which enables a 24-bit resolution and a sample rate of $100 \mathrm{kHz}$. For the BAM6QT experiments, they were replaced by two full scale stopped XCQ-062 A-screen sensors with a 100-kilopascal range.

In $\mathrm{H} 2 \mathrm{~K}$, the surface temperature on the PEEK segments is captured via two infrared cameras. The top view is captured with an AGEMA ${ }^{\circledR}$ THV570 at a sample rate of $50 \mathrm{~Hz}$ and a resolution of $320 \times 240$ pixel. The side view is captured with a FLIR ${ }^{\circledR}$ SC3000 at $60 \mathrm{~Hz}$ and a resolution of $320 \times 240$ pixel. In both facilities, the model is at room temperature $(\approx 295 \mathrm{~K})$ before the test. Since the sensor data used in this paper were captured within the first second after wind tunnel start in BAM6QT and within the first two seconds after wind

Table 2 Model dimensions and sensor positions given by the local model radius $r$ as well as the $x$-coordinate and the path length $s$ measured from the nose tip

\begin{tabular}{cccc}
\hline No. & $r, \mathrm{~mm}$ & $x, \mathrm{~mm}$ & $s, \mathrm{~mm}$ \\
\hline Parting & 15.5 & 295.8 & 296.2 \\
\hline A & 17.9 & 340.8 & 341.2 \\
B & 28.7 & 547.3 & 548.0 \\
\hline Parting & 39.5 & 753.8 & 754.8 \\
\hline 1 \& C & 40.0 & 763.8 & 764.8 \\
2 & 42.4 & 808.8 & 809.9 \\
3 & 44.7 & 853.8 & 854.9 \\
\hline Base short & 45.3 & 863.8 & 863.2 \\
\hline 4 & 47.1 & 898.8 & 900.0 \\
5 \& D & 49.5 & 943.8 & 945.1 \\
\hline Base middle & 50.5 & 963.8 & 965.1 \\
\hline E & 59.4 & 1133.8 & 1135.3 \\
\hline Base long & 60.2 & 1148.8 & 1150.3 \\
\hline
\end{tabular}


tunnel start in $\mathrm{H} 2 \mathrm{~K}$, the increase of the wall temperature is a few kelvin in laminar regions and not more than $10 \mathrm{~K}$ in turbulent regions.

\subsection{Data Processing}

The averaged frequency spectra, shown in this paper, base on 500000 data points at $0.1 \mathrm{~s}$ stable flow conditions divided into 39 blocks with 25000 samples each. Adjacent blocks overlap by $50 \%$. All values are normalized with static pressure of the inflow and then each block is multiplied with the Hann function. For each block, the power spectral density is computed. The arithmetic mean of all spectra is the final result. This procedure is also known as Welch's method. Hence, the frequency spectra show root mean square values scaled with the frequency.

To resolve single turbulent spots and wave packages, the PCB data are also processed using a complex Morlet wavelet analysis $[9,10]$. The wavelet function used here is a sine function limited in time by a Gaussian distribution:

$$
\psi(t)=\frac{1}{\sqrt[4]{\pi}} e^{-t^{2} / 2} e^{i(3 / 2) \pi t} .
$$

The wavelet transforms performed in this paper are based on 5000 samples and use 2500 scales of the wavelet with the same maximum amplitude of frequency response. Therefore, the time resolution is $2 \mu \mathrm{s}$ and the frequency resolution is $1 \mathrm{kHz}$.

The raw data of the infrared cameras are transferred to heat fluxes and Stanton numbers using the in-house tool VisualHeatFlow (for the algorithm, see [11]). The recovery factor for the postprocessing of the infrared images is always set to the value of a laminar boundary layer $\sqrt{\operatorname{Pr}}=\sqrt{0.73}$. The used coordinate system has its origin at the tip of a perfect sharp cone with the $x$-axis pointing in flow direction and the $z$-axis to the top of the test section.

\subsection{Model Alignment}

The model support in $\mathrm{H} 2 \mathrm{~K}$ allows adjustment of the pitch and yaw angles. A correct alignment results in a symmetric transition region on the cone surface. The two infrared cameras with top and side view allow the correction of the yaw and pitch angle. Figure 6 shows the alignment procedure in $\mathrm{H} 2 \mathrm{~K}$.

As there is no rotatable model support in the BAM6QT, the sting was designed to allow small corrections of the yaw and the pitch angles. Since infrared thermography is not available and the TSP is only available on one side, the signal of the PCB sensors was used to check the alignment. If 4 PCBs around 


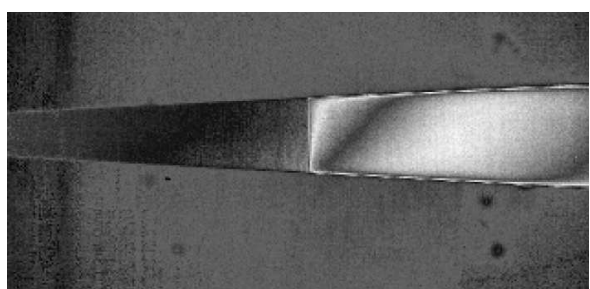

(a)

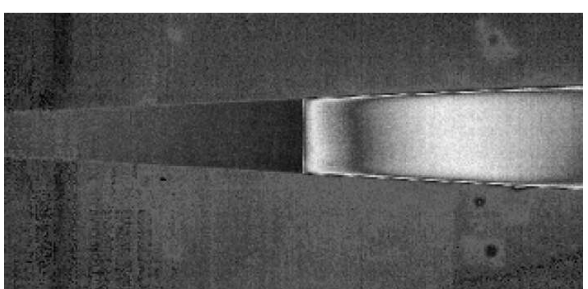

(b)

Figure 6 Model alignment in H2K: top view infrared images of the cone with ceramic middle and PEEK rear segment: $(a)$ model with 0.08 degree yaw angle; and $(b)$ model correctly aligned

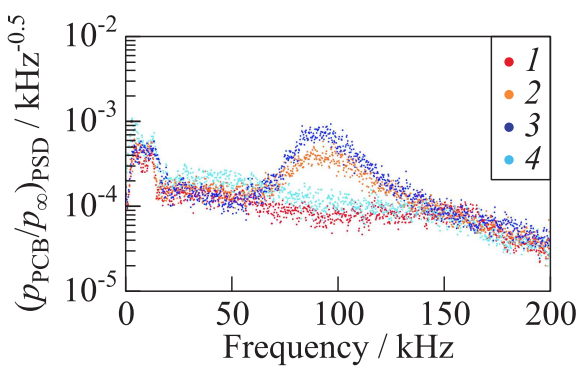

(a)

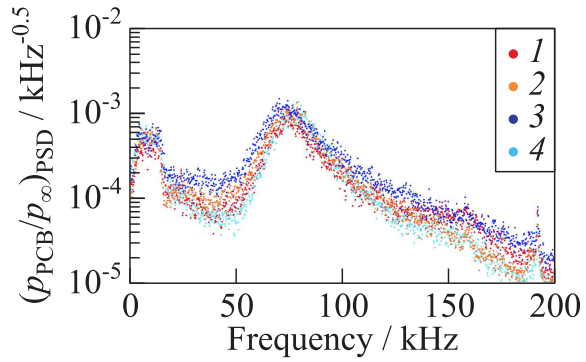

(b)

Figure 7 Model alignment in BAM6QT: signals of the 4 PCBs at position 1: (a) model with small nonzero yaw and pitch angles; $(b)$ model correctly aligned; $1-$ top; 2 - bottom; 3 - right; and 4 - left

the circumference at the same $x$ position measure the same amplitude of the second (Mack) mode, the model is correctly aligned. Figure 7 shows the alignment procedure in BAM6QT.

\section{RESULTS}

For all three inflow noise levels - BAM6QT in "noisy" mode, H2K, and BAM6QT in "quiet" mode, the second (Mack) modes were observed on the cone with plain surface. Figures 8-10 show the plots of the power spectral density computed from the PCB data in different runs. Good to see is the formation, amplification, and decay of the second (Mack) modes. It is important to note that the decay of the modes and, hence, a transition to a turbulent boundary layer was observed in quiet flow, too, even though usually a compression cone is used to obtain free transition in quiet flow in BAM6QT. 


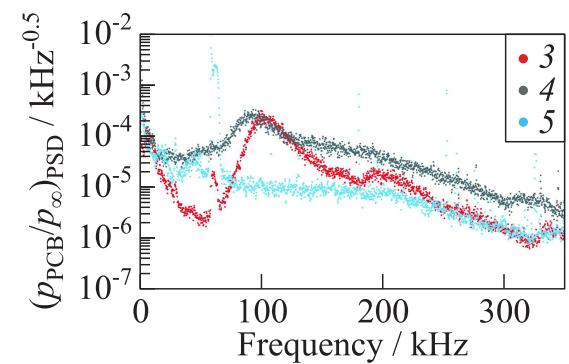

(a)

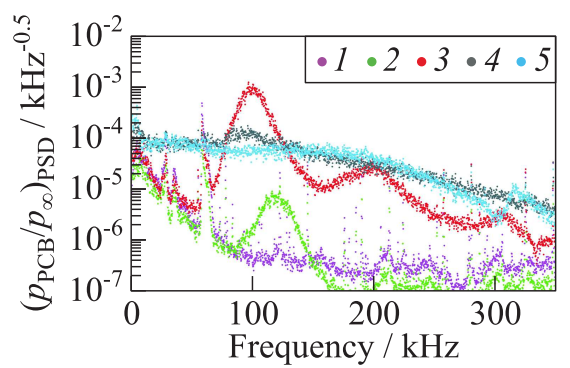

(b)

Figure 8 The second (Mack) modes on the plain model in H2K: (a) $\operatorname{Re}_{u, \infty}$ $=3.2 \cdot 10^{6} \mathrm{~m}^{-1} ;(b) \operatorname{Re}_{u, \infty}=4.1 \cdot 10^{6} \mathrm{~m}^{-1} ; 1$ - sensor at $s=341 \mathrm{~mm} ; 2$ $548 ; 3-765 ; 4-945$; and $5-$ sensor at $s=1135 \mathrm{~mm}$

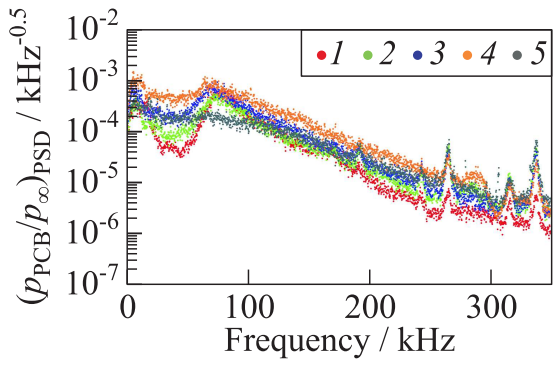

(a)

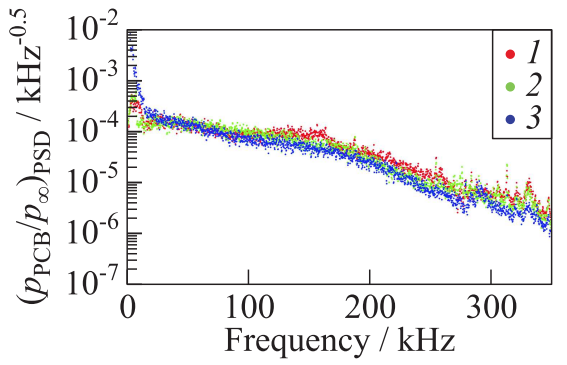

(b)

Figure 9 The second (Mack) modes on the plain model in BAM6QT with noisy flow: (a) $\operatorname{Re}_{u, \infty}=2.4 \cdot 10^{6} \mathrm{~m}^{-1} ;(b) \operatorname{Re}_{u, \infty}=3.3 \cdot 10^{6} \mathrm{~m}^{-1} ; 1$ - sensor at $s=765 \mathrm{~mm} ; 2-$ $810 ; 3-855 ; 4-900$; and $5-$ sensor at $s=945 \mathrm{~mm}$

In BAM6QT in noisy mode at $\operatorname{Re}_{u, \infty}=2.4 \cdot 10^{6} \mathrm{~m}^{-1}$, the second (Mack) mode is detected at a frequency of about $70 \mathrm{kHz}$. It disappears between the sensors at $s=900$ and $945 \mathrm{~mm}$ (see Fig. $9 c$ ) which results in a Reynolds number of about $2.2 \cdot 10^{6}$ for the completion of the transition process. In $\mathrm{H} 2 \mathrm{~K}$, at $\operatorname{Re}_{u, \infty}$ $=3.2 \cdot 10^{6} \mathrm{~m}^{-1}$, the second (Mack) mode is detected at a frequency of about $100 \mathrm{kHz}$. The transition process is completed between the sensors at $s=945$ and $1135 \mathrm{~mm}$ (see Fig. 8a) which results in a Reynolds number of about $3.2 \cdot 10^{6} \mathrm{~m}^{-1}$. At $\operatorname{Re}_{u, \infty}=4.1 \cdot 10^{6} \mathrm{~m}^{-1}$, a sensor at $s=765 \mathrm{~mm}$ also measures the first and second harmonic of the second (Mack) mode (see Fig. $8 b$ ). In the BAM6QT in quiet mode at $\operatorname{Re}_{u, \infty}=11.5 \cdot 10^{6} \mathrm{~m}^{-1}$, the second (Mack) mode is detected at a frequency of about $140 \mathrm{kHz}$. The transition process is almost completed at the sensor at $s=945 \mathrm{~mm}$ (see Fig. $10 \mathrm{~d}$ ) which results in 


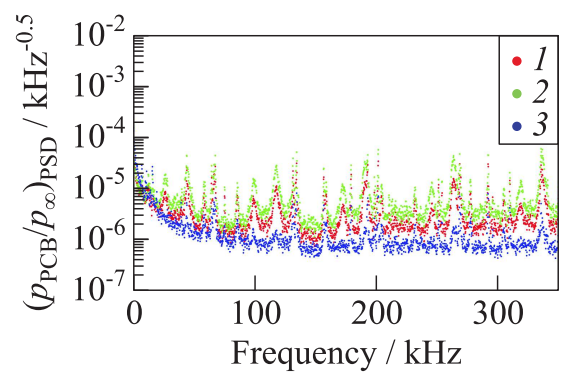

(a)

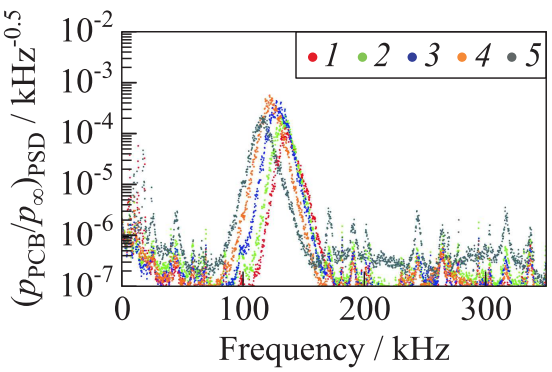

(c)

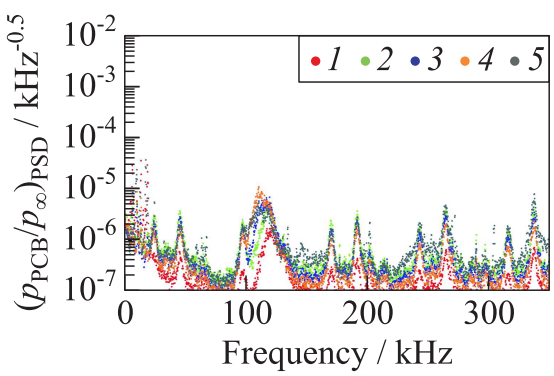

(b)

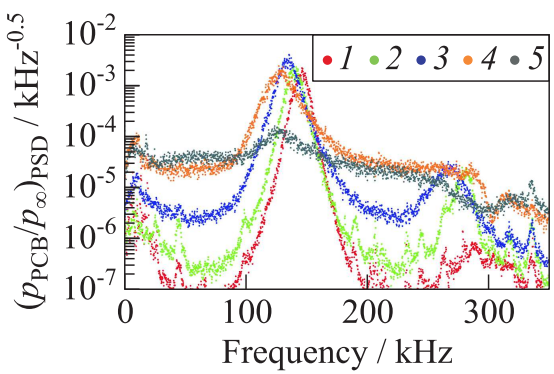

(d)

Figure 10 The second (Mack) modes on the plain model in BAM6QT with quiet flow: $(a) \operatorname{Re}_{u, \infty}=2.3 \cdot 10^{6} \mathrm{~m}^{-1}$; (b) $7.2 \cdot 10^{6} ;$ (c) $9.5 \cdot 10^{6}$; (d) $\operatorname{Re}_{u, \infty}=11.5 \cdot 10^{6} \mathrm{~m}^{-1}$; 1 - sensor at $s=765 \mathrm{~mm} ; 2-810 ; 3-855 ; 4-900$; and $5-$ sensor at $s=945 \mathrm{~mm}$

a Reynolds number of about $11.0 \cdot 10^{6} \mathrm{~m}^{-1}$. The sensors at $s=810$ and $855 \mathrm{~mm}$ also measure the first harmonic of the second (Mack) mode. Besides the shift to lower Reynolds numbers with increasing turbulent intensity of the inflow, there is no principal difference in the transition process observed. Also, the maximum of the normalized amplitude of the second (Mack) mode is of the same order of magnitude. This supports the attempt of Marineau et al. [12] of an amplitudebased method to account for the effect of tunnel noise on the second (Mack) mode transition.

This is supported by the wavelet plots in Figs. 11 to 13 . Figure 11 shows the wavelet analysis of data from $\mathrm{H} 2 \mathrm{~K}$ at $\operatorname{Re}_{u, \infty}=4.1 \cdot 10^{6} \mathrm{~m}^{-1}$. The corresponding spectra can be found in Fig. $8 b$. At $s=341.2 \mathrm{~mm}$, the boundary layer is still laminar and the wavelet plot of the sensor data reveals no interesting features (Fig. 11a). At $s=548.0 \mathrm{~mm}$, the first compact packages of the second (Mack) modes occur that indicate a transitional boundary layer (Fig. 11b). They are limited in time and frequency. They increase in amplitude and number until $s=764.8 \mathrm{~mm}$ (Fig. 11c). At $s=945.1 \mathrm{~mm}$, the compact packages are destroyed 


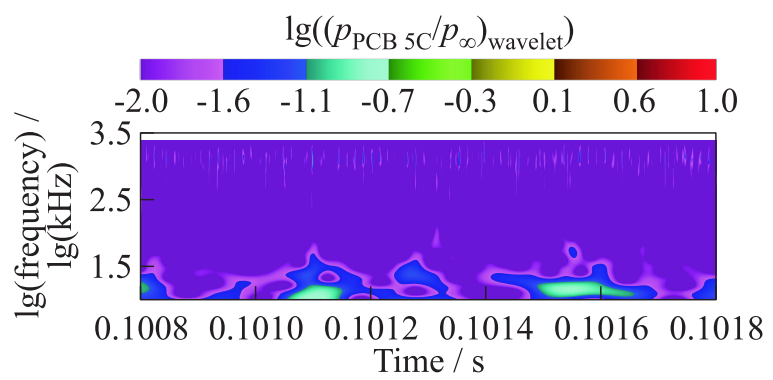

(a)

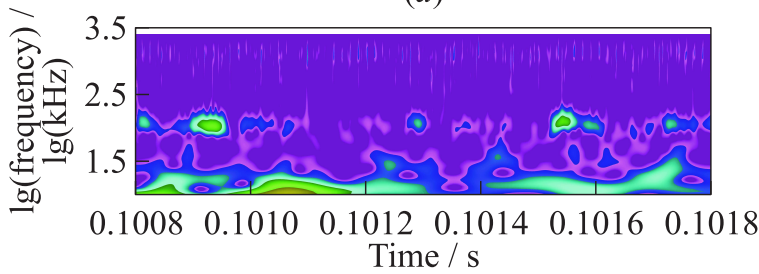

(b)

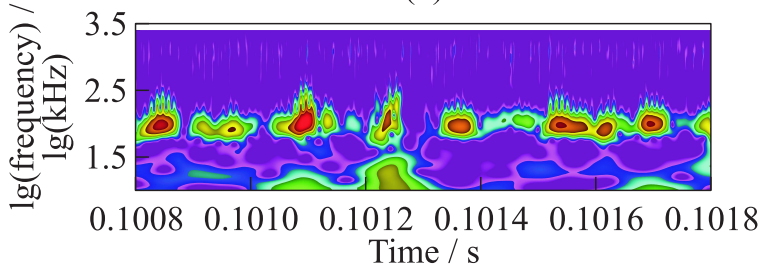

(c)

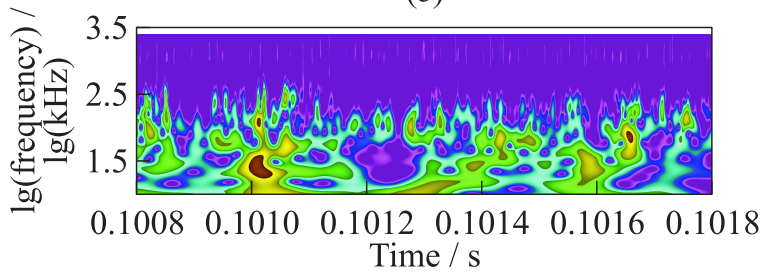

(d)

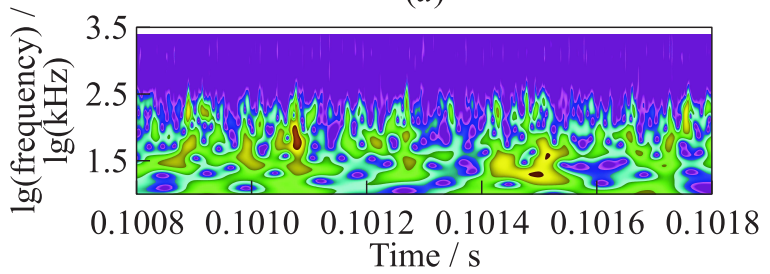

(e)

Figure 11 Wavelets in $\mathrm{H} 2 \mathrm{~K}$ at $\operatorname{Re}_{u, \infty}=4.1 \cdot 10^{6} \mathrm{~m}^{-1}$ : (a) sensor at $s=341.2 \mathrm{~mm}$; (b) $548.0 ;(c) 764.8 ;(d) 945.1$; and $(e)$ sensor at $s=1135.3 \mathrm{~mm}$ 


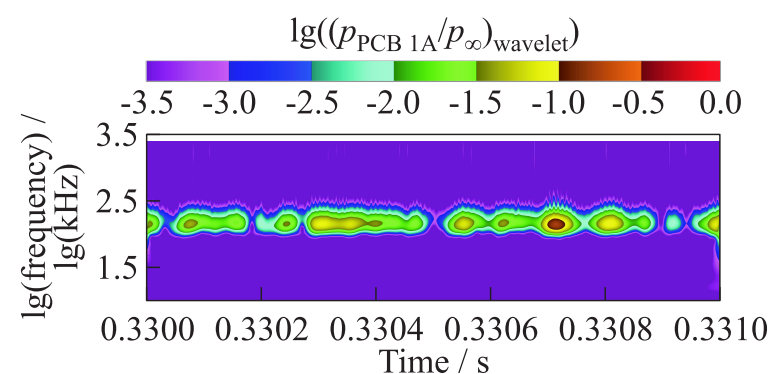

(a)

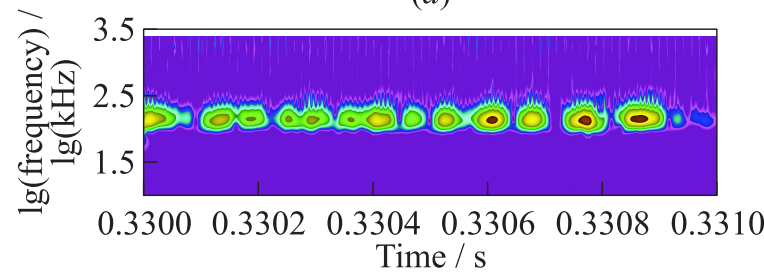

(b)

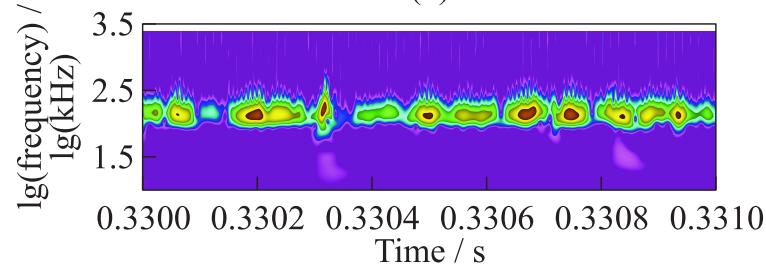

(c)

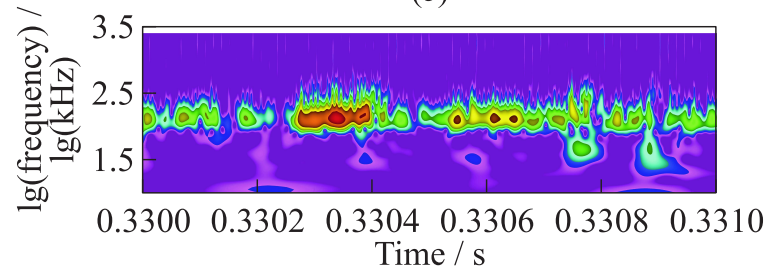

(d)

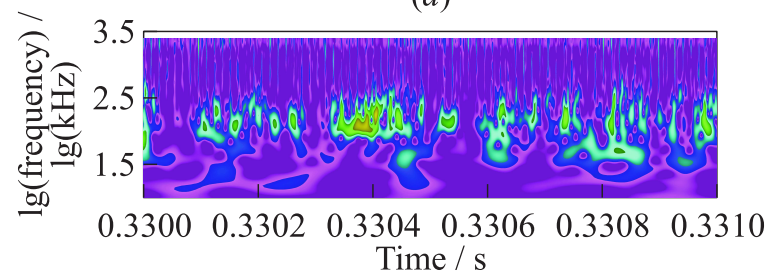

(e)

Figure 12 Wavelets in BAM6QT with quiet flow at $\operatorname{Re}_{u, \infty}=11.5 \cdot 10^{6} \mathrm{~m}^{-1}:(a)$ sensor at $s=764.8 \mathrm{~mm} ;(b) 809.9 ;(c) 854.9 ;(d) 900.0$; and $(e)$ sensor at $s=945.1 \mathrm{~mm}$ 


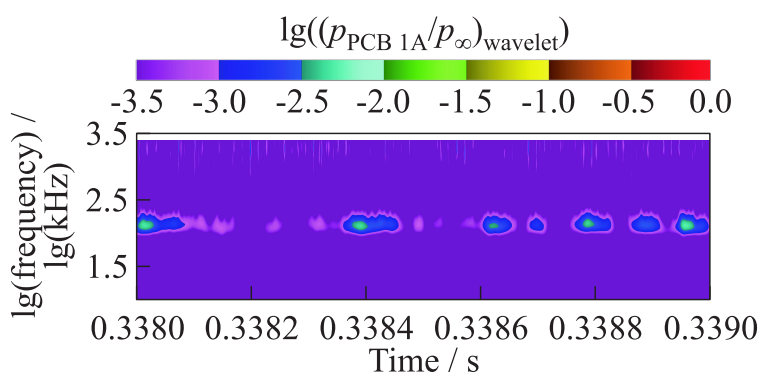

(a)

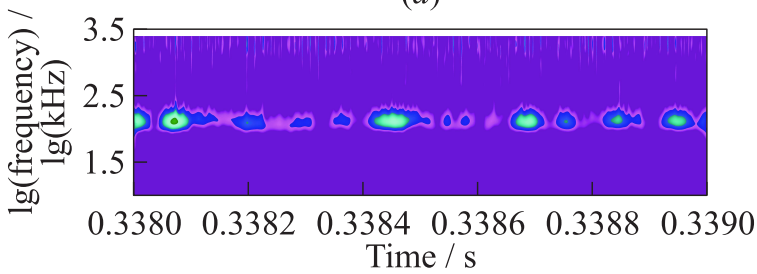

(b)

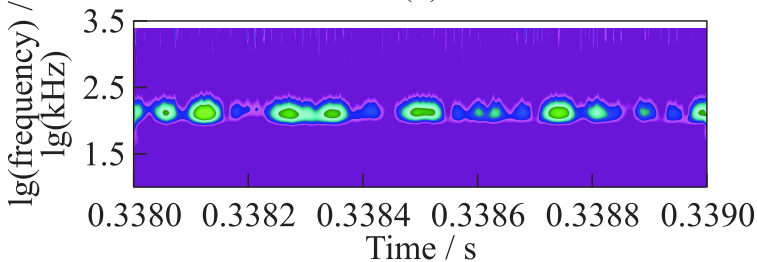

(c)

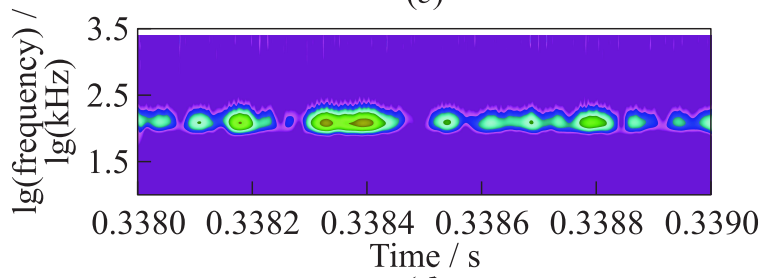

(d)

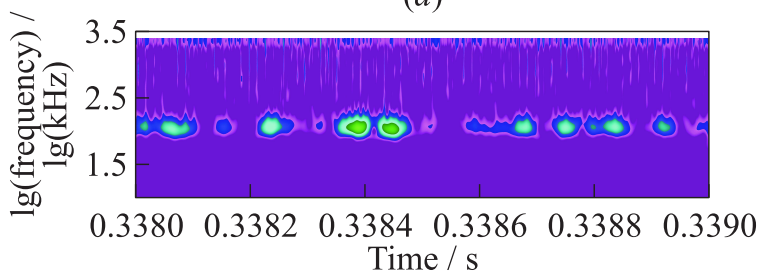

(e)

Figure 13 Wavelets in BAM6QT with quiet flow at $\operatorname{Re}_{u, \infty}=9.5 \cdot 10^{6} \mathrm{~m}^{-1}:(a)$ sensor at $s=764.8 \mathrm{~mm}$; $(b) 809.9 ;(c) 854.9 ;(d) 900.0$; and (e) sensor at $s=945.1 \mathrm{~mm}$ 
and spread in time and frequency (Fig. 11d). There are no distinguishable packages at $s=1135.3 \mathrm{~mm}$ but a vesicular structure covering the complete time span and a broad frequency band - the boundary layer is fully turbulent (Fig. 11e). This is consistent with the measurements of free transition on a flat panel at 0 degree angle of attack as shown in [13].

The compact packages and their decay are also detected in BAM6QT at $\operatorname{Re}_{u, \infty}=11.5 \cdot 10^{6} \mathrm{~m}^{-1}$ as shown in Fig. 12. But at the last sensor, there are still distinguishable packages. This indicates, that the transition process is not fully completed. In the wavelet analysis of data at $\operatorname{Re}_{u, \infty}=9.5 \cdot 10^{6} \mathrm{~m}^{-1}$, the same packages are visible on different sensors (see Fig. 13). This allows an estimation of the travelling speed of these package to $900 \mathrm{~m} / \mathrm{s}$ which is close to the computed edge velocity of $850 \mathrm{~m} / \mathrm{s}$.

The comparison of the transition process with ultrasonically absorptive materials shows no damping of the second (Mack) mode or transition delay, neither in noisy nor in quiet flow.

Figures 14 and 15 show the results from $\mathrm{H} 2 \mathrm{~K}$ at $\operatorname{Re}_{u, \infty}=4.1 \cdot 10^{6} \mathrm{~m}^{-1}$ and from BAM6QT at $\operatorname{Re}_{u, \infty}=2.4 \cdot 10^{6} \mathrm{~m}^{-1}$ in noisy flow, respectively. There is just

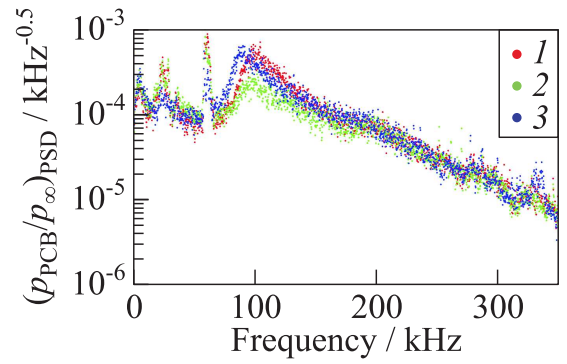

(a)

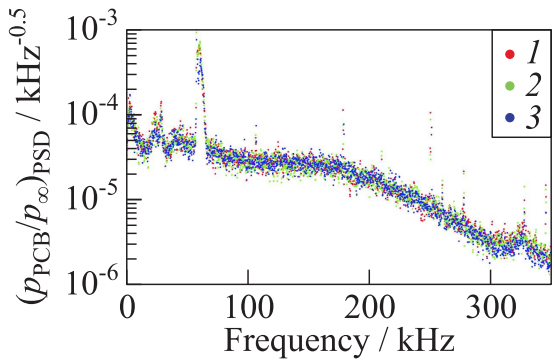

(b)

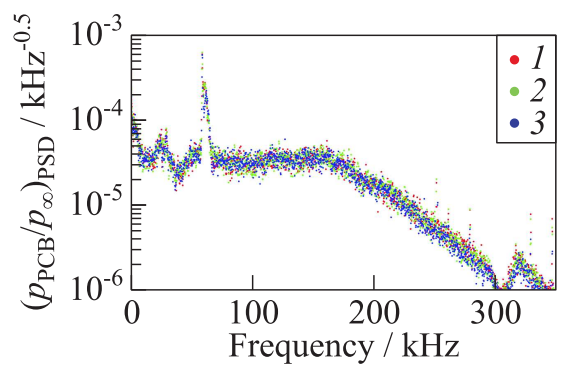

(c)

Figure 14 The second (Mack) modes on different surfaces $(1-$ plain; 2 - regular holes; and 3 - random pores) in $\mathrm{H} 2 \mathrm{~K}$ at $\operatorname{Re}_{u, \infty}=4.1 \cdot 10^{6} \mathrm{~m}^{-1}$ : (a) sensor at $s=765 \mathrm{~mm}$; (b) 945 ; and (c) sensor at $s=1135 \mathrm{~mm}$ 


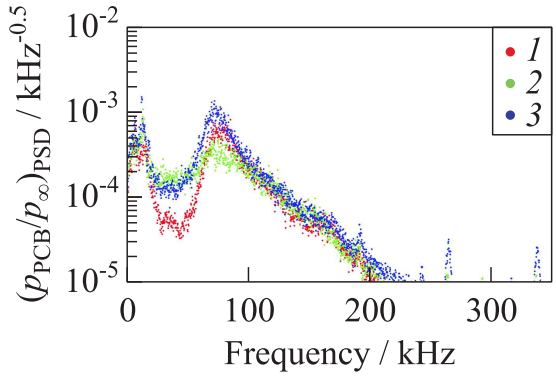

(a)

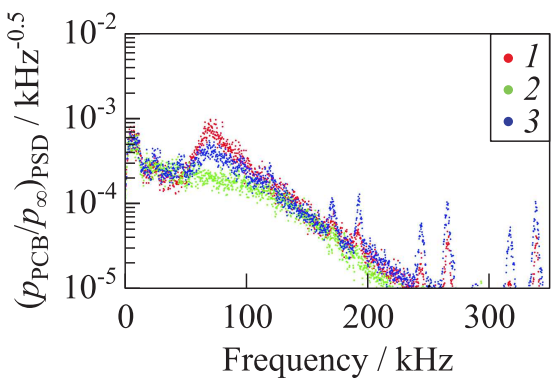

(c)

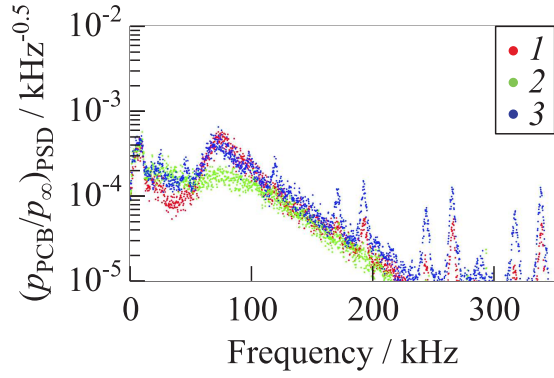

(b)

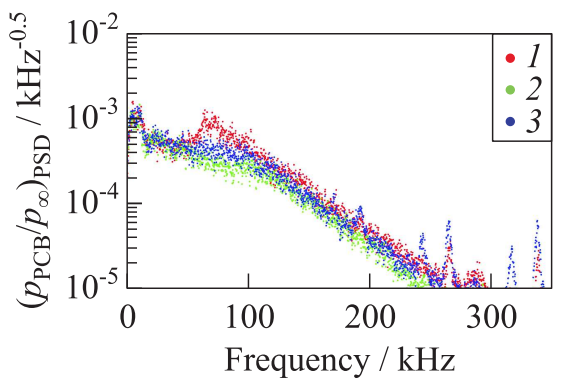

(d)

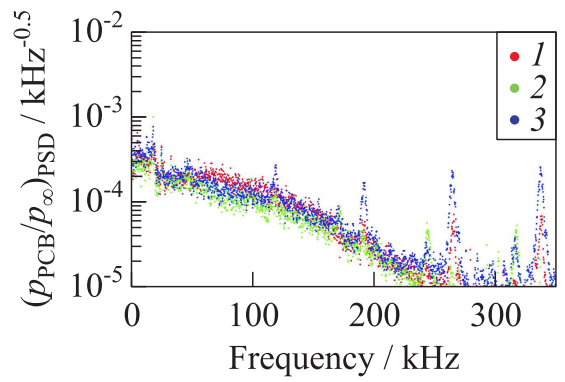

(e)

Figure 15 The second (Mack) modes on different surfaces $(1-$ plain; 2 - regular holes; and 3 - random pores) in BAM6QT with noisy flow at $\operatorname{Re}_{u, \infty}=4.1 \cdot 10^{6} \mathrm{~m}^{-1}$ : (a) sensor at $s=765 \mathrm{~mm}$; (b) 810; (c) 855; (d) 900; and (e) sensor at $s=945 \mathrm{~mm}$ 


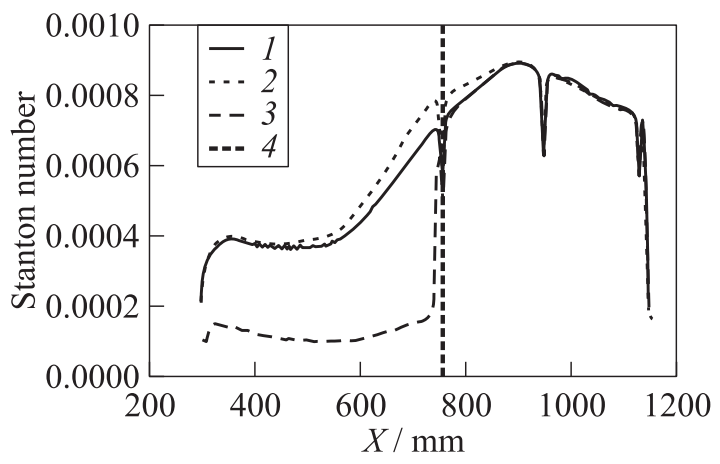

Figure 16 Results of infrared thermography in $\mathrm{H} 2 \mathrm{~K}$ at $\operatorname{Re}_{u, \infty}=4.1 \cdot 10^{6} \mathrm{~m}^{-1}: 1$ plain surface; 2 - holes; 3 - ceramic surface; and 4 - PCB C

a small difference in the amplitudes on the different materials, but the second (Mack) mode on the regular holes decay earlier than on the other materials, which even indicates an earlier transition there. The second (Mack) mode on the random pores decays just a little earlier than on the plain surface. This is consistent with the results of the infrared thermography made in $\mathrm{H} 2 \mathrm{~K}$ that show a slight shift of the transition region (indicated by a raise of the Stanton number) on the regular holes upstream (Fig. 16).

Figures 17 and 18 show the results from BAM6QT at $\operatorname{Re}_{u, \infty}=9.5 \cdot 10^{6}$ and $11.5 \cdot 10^{6} \mathrm{~m}^{-1}$ in quiet flow. At $\operatorname{Re}_{u, \infty}=9.5 \cdot 1-{ }^{6} \mathrm{~m}^{-1}$, all sensors on the rear part detect strong second (Mack) modes (see Fig. 17). All figures there show an amplification of the second (Mack) mode and a shift to lower frequencies on the regular holes and on the random pores compared to the plain surface. At $\operatorname{Re}_{u, \infty}=11.5 \cdot 10^{6} \mathrm{~m}^{-1}$, the decay of the second (Mack) mode on the rear segment indicates the end of the transition region (see Fig. 18). The figures there show an earlier decay of the second (Mack) mode and a shift to lower frequencies on the regular holes compared to the plain surface. With the random pores, the amplitudes are little higher at the first sensors compared to the plain surface. And the decay of the second (Mack) mode is a little faster on the last sensors.

\section{SUMMARY}

The experiments with the same sharp slender cone and the same data acquisition system carried out in the quiet wind tunnel BAM6QT and the conventional wind tunnel $\mathrm{H} 2 \mathrm{~K}$ at similar conditions at Mach 6 allow direct comparison of the laminar-turbulent transition of a hypersonic boundary layer. Due to the small half angle of $3^{\circ}$ and the large model length, the amplification and breakdown of 


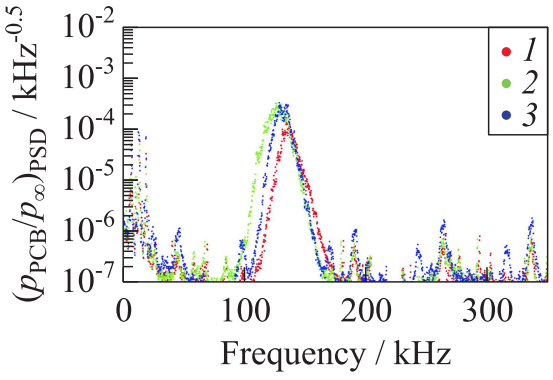

(a)

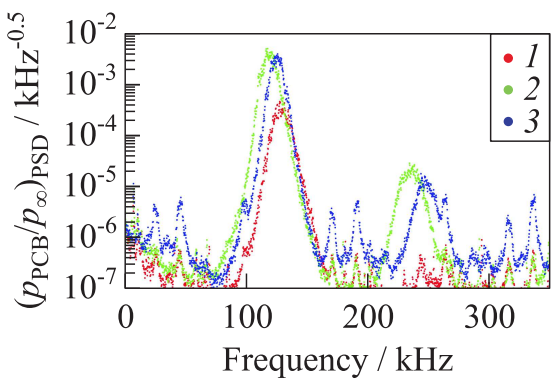

(c)

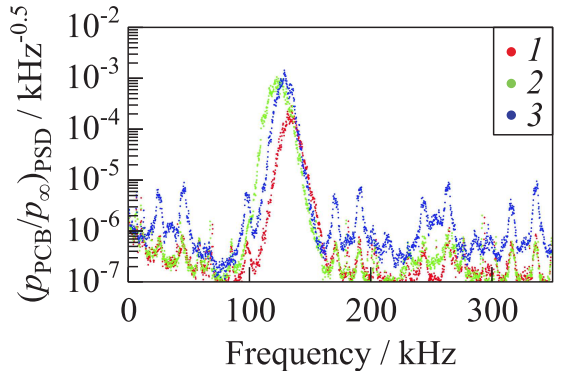

(b)

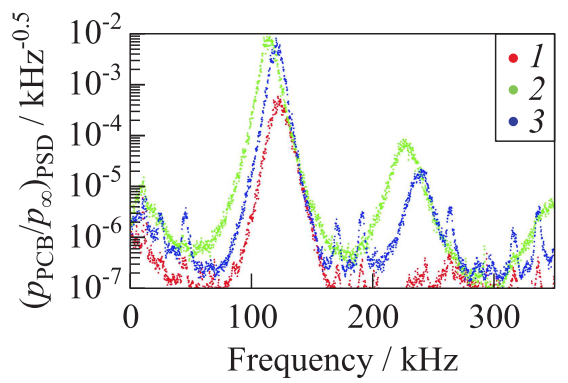

(d)

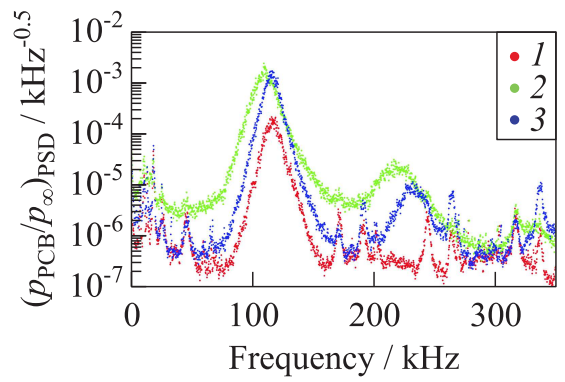

(e)

Figure 17 The second (Mack) modes on different surfaces $(1-$ plain; 2 - regular holes; and 3 - random pores) in BAM6QT with quiet flow at $\operatorname{Re}_{u, \infty}=9.5 \cdot 10^{6} \mathrm{~m}^{-1}$ : (a) sensor at $s=765 \mathrm{~mm}$; (b) 810; (c) 855; (d) 900; and (e) sensor at $s=945 \mathrm{~mm}$ 


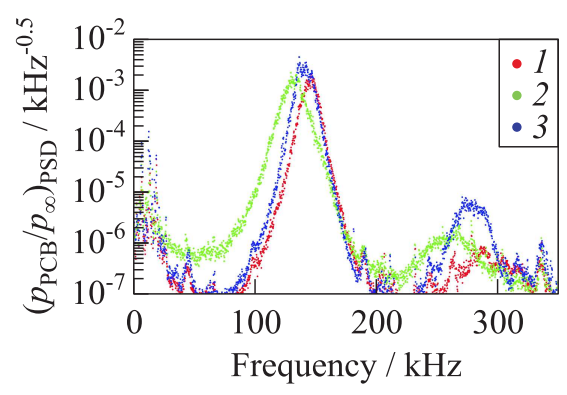

(a)

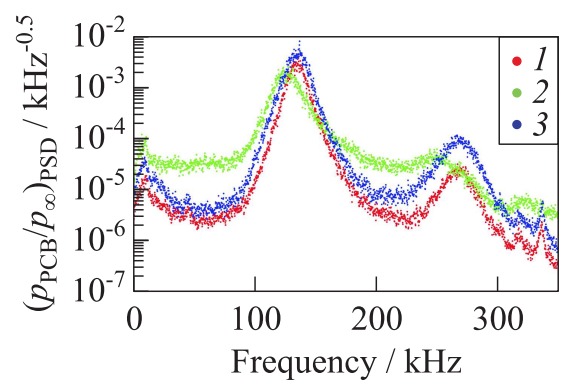

(c)

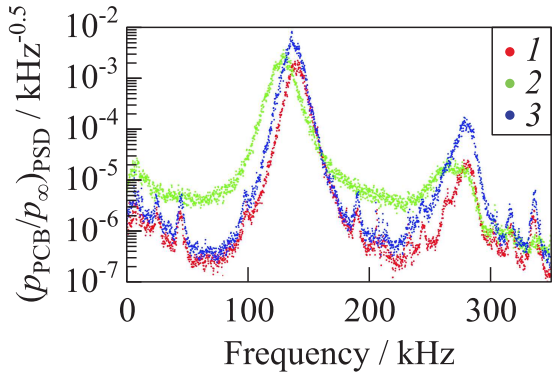

(b)

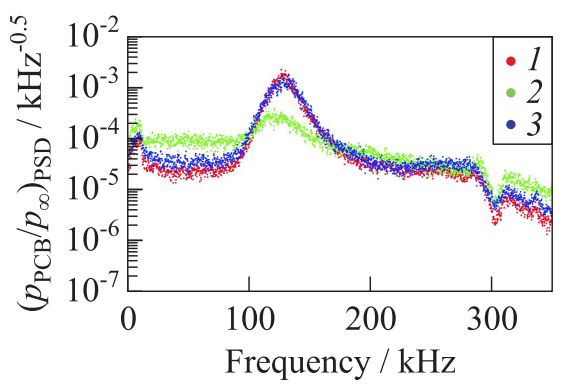

(d)

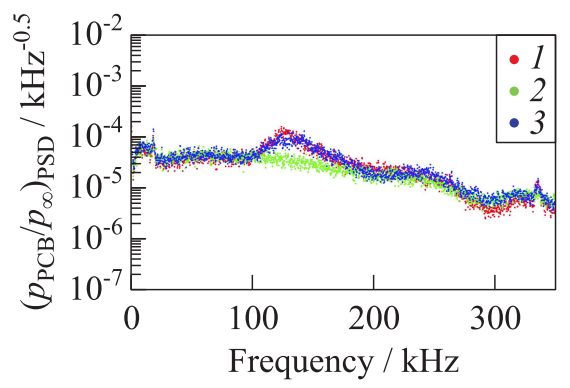

(e)

Figure 18 The second (Mack) modes on different surfaces $(1-$ plain; 2 - regular holes; and 3 - random pores) in BAM6QT with quiet flow at $\operatorname{Re}_{u, \infty}=11.5 \cdot 10^{6} \mathrm{~m}^{-1}$ : (a) sensor at $s=765 \mathrm{~mm}$; $(b) 810 ;(c) 855$; (d) 900; and (e) sensor at $s=945 \mathrm{~mm}$ 
the second (Mack) modes could be observed for noisy as well as for quiet conditions. While there are evident differences in the transition locations between quiet and noisy conditions, the transition process and mechanism seem to be similar.

To test the damping effect of porous surfaces, two alternative sections were used, one with regular holes and the other with random pores. The experiments performed in the BAM6QT under noisy and quiet conditions and in $\mathrm{H} 2 \mathrm{~K}$ provided similar results. Although the design of the holes and pores was based on numerical simulations, neither a significant damping of the second (Mack) mode nor a delay of the transition process occurred. On the contrary, the results indicate an amplification of the second (Mack) mode and an earlier transition, especially on the regular holes. The reason for this discrepancy is not yet known. A possible explanation are the lower frequencies of the second (Mack) mode on a 3 degree cone compared to a 5 degree cone used by Rasheed et al. [1] or to 7 degree cones used by Fedorov et al. [2] and Wagner et al. [3]. Another reason could be the sensitivity of the damping to the position and length of the porous surfaces as found by Lukashevich et al. [14].

\section{REFERENCES}

1. Rasheed, A., H. G. Hornung, A. V. Fedorov, and N. D. Malmuth. 2002. Experiments on passive hypervelocity boundary-layer control using an ultrasonically absorptive surface. AIAA J. 40(3):481-489.

2. Fedorov, A. V., A. N. Shiplyuk, A. A. Maslov, E. V. Burov, and N. D. Malmuth. 2003. Stabilization of a hypersonic boundary layer using an ultrasonically absorptive coating. J. Fluid Mech. 479:99-124.

3. Wagner, A., M. Kuhn, J. M. Schramm, and K. Hannemann. 2013. Experiments on passive hypersonic boundary layer control using ultrasonically absorptive carboncarbon material with random microstructure. Exp. Fluids 54(10):1606.

4. Wartemann, V., H. Lüdeke, and N. D. Sandham. 2009. Stability analysis of hypersonic boundary layer flow over microporous surfaces. AIAA Paper No. 2009-7202. $10 \mathrm{p}$.

5. Wartemann, V., and H. Lüdeke. 2010. Investigation of slip boundary conditions of hypersonic flow over microporous surfaces. European Conference on Computational Fluid Dynamics, ECCOMAS CFD. Eds. Pereira, J. C. F., and A. Sequeira. Lisbon: ECCOMAS. 1-12.

6. Walther, K., M. Brajdic, I. Kelbassa, and R. Poprawe. 2008. Bohren mit gepulster Laserstrahlung. wt Werkstattstechnik online 98(6):520-523.

7. Chynoweth, B. C., C. A. C. Ward, R. O. Henderson, C. G. Moraru, R. T. Greenwood, A.D. Abney, and S. P. Schneider. 2014. Transition and instability measurements in a Mach 6 quiet wind tunnel. AIAA Paper No. 2014-0074. 
8. Niezgodka, F.-J. 2001. Der Hyperschallwindkanal H2K des DLR in Köln-Porz (Stand 2000). Köln: DLR-Mitteilungen. Deutsches Zentrum für Luft- und Raumfahrt e. V.

9. Grossman, A., and J. Morlet. 1985. Decomposition of functions into wavelets of constant shape, and related transforms. Mathematics and physics: Lectures on recent results. Ed. L. Streit. Singapore: World Scientific. 135-165.

10. Bentley, P. M. and J. T. E. McDonnel. 1994. Wavelet transforms: An introduction. Electron. Commun. Eng. J. 6(4):175-186. doi: 10.1049/ecej:19940401.

11. Henckels, A., and P. Gruhn. 2004. Study on aerothermal effects of viscous shock interaction in hypersonic inlets. 5th European Symposium on Aerothermodynamics for Space Vehicles Proceedings. Cologne: European Space Agency. 553-558.

12. Marineau, E. C., G. C. Moraru, D. R. Lewis, J. D. Norris, J. F. Lafferty, R. M. Wagnild, and J.A. Smith. 2014. Mach 10 boundary layer transition experiments on sharp and blunted cones. AIAA Paper No. 2014-3108. 24 p.

13. Willems, S., A. Gülhan, and J. Steelant. 2015. Experiments on the effect of laminarturbulent transition on the SWBLI in H2K at Mach 6. Exp. Fluids 56(3):49.

14. Lukashevich, S. V., S. O. Morozov, and A. N. Shiplyuk. 2013. Experimental study of the effect of a passive porous coating on disturbances in a hypersonic boundary layer. 1. Effect of the porous coating length. J. Appl. Mech. Tech. Phys. 54(4):572577. 